\title{
Hausärzte und Forschung - geht das zusammen?
}

\author{
Forschungsinstitute für Hausarztmedizin sind auf die Zusammenarbeit mit Haus- \\ ärzten angewiesen, denn nur so haben sie Zugang zu Patienten - anders als die \\ Forschung in klinischen Einrichtungen. Karrierefördernd ist Forschung für Haus- \\ ärzte allerdings nicht. Sind sie trotzdem bereit, sich zu engagieren? Müsste ihr \\ Engagement in der Forschung auch abgegolten werden?
}

Thomas Rosemann

Korrespondenz:

Prof. Dr. Thomas Rosemann PhD Institutsdirektor Institut für Hausarztmedizin

UniversitätsSpital Zürich Pestalozzistrasse 24 CH-8091 Zürich

thomas.rosemann[at]usz.ch

\section{Forschung als Grundbedingung für die Akademisierung der Hausarztmedizin} Eine zentrale Forderung der Volksinitiative zur Hausarztmedizin ist die Akademisierung der Hausarztmedizin, also die akademische Vertretung durch Lehrstühle an allen Schweizer Universitäten. Forschung ist dabei ein zentraler Aspekt, ist sie doch ein Charakteristikum einer jeden eigenständigen Profession. Dabei ist Forschung kein akademischer Selbstzweck, sondern dient einerseits der Beschreibung (und damit auch Definition) des Fachgebietes, andererseits aber vor allem auch der Weiterentwicklung des Fachs bzw. der im Fach eingesetzten diagnostischen und therapeutischen Instrumente, bis hin zur Weiterentwicklung des gesamten Versorgungsgefüges.

Im Gegensatz zu einer regulären klinischen Einrichtung verfügen die Institute für Hausarztmedizin in der Regel nicht über einen direkten Zugang zu Patienten. In der Forschung sind sie also auf die Zusammenarbeit mit den klinischen/praktischen Vertretern ihres Fachs, also den Hausärzten, angewiesen. Auch hier gibt es einen fundamentalen Unterschied zu universitären Kliniken, denn während in den Kliniken Forschung oft durch intrinsisch motivierten Nachwuchs erfolgt, der damit auch an eigenen Karrierewegen bastelt, kann die Karriere in der Regel nicht die führende Motivation für Hausärzte sein, sich an Forschungsprojekten zu beteiligen. Hausärzte für Forschungsprojekte zu gewinnen, ist daher in der Hausarztmedizin ungleich schwieriger als in anderen klinischen Fächern: Hausärzte sind auch selbständige Unternehmer mit berechtigten finanziellen Interessen und wirtschaftlichem Druck, dem klinische Forscher in dieser Weise nicht unterstehen.

\section{Was motiviert Hausärzte, sich an Forschung zu beteiligen?}

Es stellt sich also die berechtigte Frage, ob sich in der Schweiz überhaupt ausreichend Hausärzte finden, die bereit sind, sich an Forschungsprojekten zu beteiligen, oder ob diese Grundvoraussetzung fehlt und man die Forderung nach universitären hausärztlichen Instituten nicht schon deswegen fallen

\section{Médecine de famille et recherche sont-elles compatibles?}

Une recherche indépendante portant sur des questions spécifiques à la discipline est inhérente à chaque profession et sert tant à attester les performances qu'à développer la discipline en question. La méthodologie de la recherche en médecine de famille est exigeante et fait appel à des médecins motivés et engagés. L'institut pour la médecine de famille de Zurich a lancé en $\mathbf{2 0 0 8}$ une série d'études montrant que les médecins de famille peuvent tout à fait s'impliquer dans des projets de recherche exigeants.

lassen sollte. Um dies zu beantworten, muss man sich zunächst die Frage stellen, was Hausärzte überhaupt motiviert, sich an Forschungsprojekten zu beteiligen. Untersuchungen zeigen, dass es folgende Faktoren sind [1]:

- Das Forschungsthema bzw. die Fragestellung muss als relevant für den hausärztlichen Alltag wahrgenommen werden.

- Die Studie muss unter Praxisbedingungen mit vertretbarem Aufwand für den Hausarzt durchführbar sein.

- Die Studie muss potentiell die Leistung der Hausarztmedizin valide beschreiben oder eine interessante Weiterentwicklungsmöglichkeit untersuchen.

- Ein minimales finanzielles Incentive, das zumindest eine formale Anerkennung der Beteiligung ausdrückt, sollte vorhanden sein.

Die Rekrutierungsrate ist dann am besten, wenn eine gegenseitige Rekrutierung innerhalb des Kollegenkreises (peer group) stattfindet, etwa im Rahmen von Ärztenetzen oder Qualitätszirkeln. Durch postali- 
schen Aufruf sind in der Regel nur fünf bis maximal zehn Prozent der Hausärzte zu bewegen, sich an einer Interventionsstudie zu beteiligen.

Diese Daten stammen sämtlich aus dem Ausland - wie verhält es sich nun in der Schweiz mit der Bereitschaft von Hausärzten, sich an Forschung zu beteiligen?
Patienten. Methodisch ist Forschung in der Hausarztmedizin darum oft anspruchsvoller als klinische Forschung in einem hochkontrollierten Setting.

Eine grosse Hürde stellen zudem einige Ethikkommissionen dar. Oft sind sie mit der Art der hausärztlichen Fragestellungen nicht vertraut. So ist etwa die Anforderung der Ethikkommissionen, jeder teil-

\section{Forschung ist kein akademischer Selbstzweck, sondern dient der Weiterentwicklung des Fachs und der diagnostischen und therapeutischen Instrumente.}

\section{Forschung in der Hausarztmedizin ist methodisch anspruchsvoll}

Das Institut für Hausarztmedizin in Zürich führt seit seiner Gründung 2008 zahlreiche Studien unterschiedlicher Art durch. Neben der kontinuierlichen Datensammlung im immer grösser werdenden FIRENetzwerk (www.icpc.ch) und Querschnittserhebungen, die mittels postalischer oder elektronischer Umfrager [3, 4] erfolgen, sind dies insbesondere (Cluster-)randomisierte Interventionsstudien [5-7]. Der Weg zur Interventionsstudie ist dabei ungleich steiniger als in anderen Fächern. Dabei ist die schwierige Drittmitteleinwerbung nur der erste Stein auf dem Weg. Wichtig ist, dass Studien in der Hausarztmedizin höchsten methodischen Ansprüchen genügen. Durch das komplexe Setting mit einer Fülle von Variablen und möglichen Störgrössen (Confounders) ist dies oft schon im Studienprotokoll anspruchsvoll abzubilden. Clusterdesigns, welche die Intraclusterkorrelationen berücksichtigen, erhöhen zudem oft die erforderliche Anzahl an Praxen bzw.

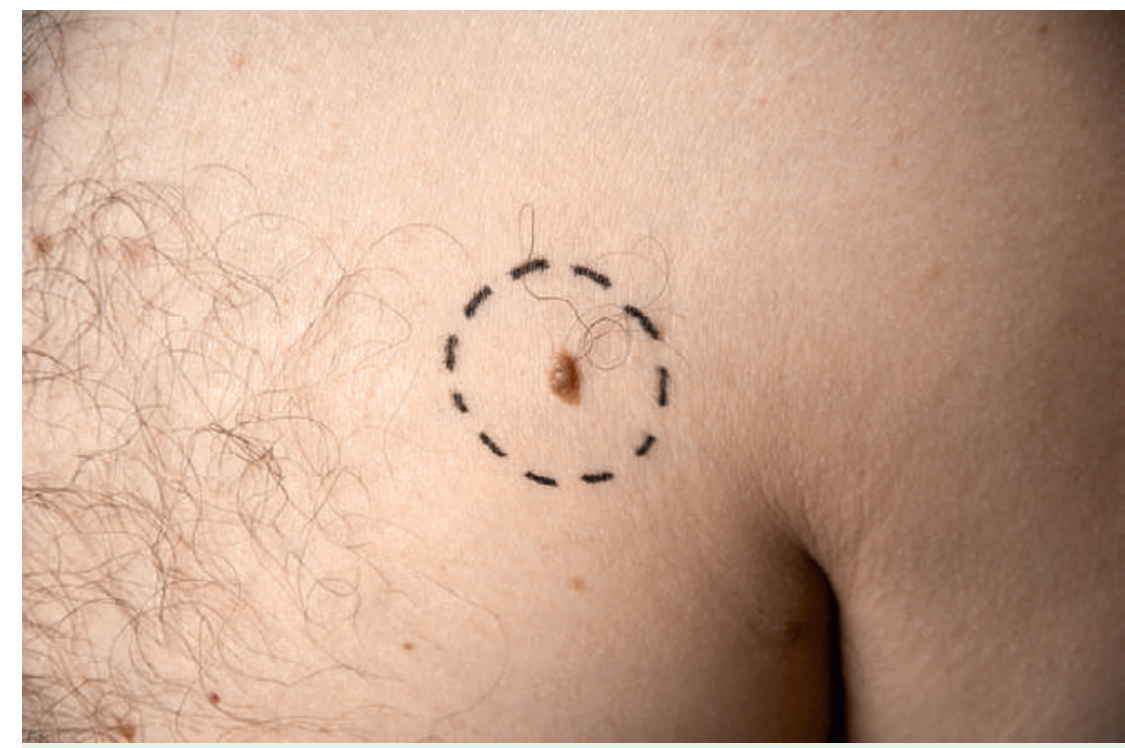

Beispiel erfolgreicher hausärztlicher Forschung: die «minSKIN-Studie» mit dem Ziel, die Fähigkeiten von Hausärzten zur Beurteilung suspekter Hautveränderungen zu optimieren. nehmende Hausarzt müsse einen Kurs in Good clinical practice absolvieren, nicht erfüllbar (und international auch absolut unüblich). Hinzu kommt, dass die meisten Studien in mehreren Kantonen durchgeführt werden, also mehrere Ethikkommissionen involviert sind. Der Gedanke der Leit-Ethikkommission, die das Studienprotokoll zuerst evaluiert und der sich die übrigen kantonalen Ethikkommissionen in einem verkürzten Zweitbeurteilungsverfahren anschliessen, bleibt oft Wunschdenken, wie zahlreiche individuelle Änderungswünsche zeigen. Im Institut für Hausarztmedizin (IHAM) Zürich ist es üblich, alle Studien vor der Einreichung bei der Ethikkommission einem internationalen Peer-Review zu unterziehen, die Studie im internationalen Trial Register $\mathrm{zu}$ registrieren und das ausführliche Protokoll frei verfügbar zu publizieren.

\section{minSKIN- und CARAT-Studie als Beispiel erfolgreicher hausärztlicher Forschung in der Schweiz}

Zwei dieser Studien, minSKIN [6] und CARAT [7], seien hier exemplarisch skizziert. minSKIN ist eine Studie, die gemeinsam mit der Dermatologischen Klinik des USZ durchgeführt wird. Ziel ist es dabei, die diagnostischen Fähigkeiten des Hausarztes in Bezug auf maligne Hautveränderungen weiter zu verbessern. Dazu wurden 70 Hausärzte in zwei Studienarme randomisiert. In der Interventionsgruppe wurden neben fallbasierten Schulungen Auflichtmikroskope verteilt und Digitalkameras. Mit diesen Kameras können Fotos suspekter Veränderungen angefertigt werden, die dann elektronisch via IHAM an die Dermatologie weitergeleitet werden. Von dort erhält der Hausarzt eine kurze Befundung und Empfehlung für das weitere Vorgehen. Die Hypothese ist, dass dieses kontinuierliche Online-Feedback in Verbindung mit gezielten edukativen Peer-Meetings zur Verbesserung der diagnostischen Fähigkeiten (diagnostic accuracy) und zu weniger unnötigen Hautexzisionen führt. In diesem Projekt zeigt sich schön, wie die Themen «integrierte Versorgung» und «eHealth» in einem konkreten Projekt in die Routineversorgung 
implementiert werden und zudem eine klinisch fokussierte Fragestellung beantwortet wird. Im Falle einer Bejahung der Studienhypothese wäre über eine flächendeckende Ausweitung des Online-Feedbacks nachzudenken, was insbesondere für Praxen in ländlichen Regionen vorteilhaft wäre, wo der nächste Dermatologe besonders weit entfernt ist. Die Bereitschaft der Hausärzte zur Teilnahme an minSkin hat alle Erwartungen übertroffen. Wir mussten etlichen Kollegen sogar absagen, insbesondere weil die finanziellen Mittel für zusätzliche Kameras nicht vorhanden waren.

Etwas aufwendiger als in minSKIN war die Rekrutierung von Hausärzten für CARAT. In dieser Studie wird ein teamorientierter Versorgungsansatz für Diabetiker in einer clusterrandomisierten Studie untersucht. Die Interventionen basieren auf den Ergebnissen eines eigens dafür durchgeführten systematischen Reviews [8]. Konkret wurden MPAs der teilnehmenden Praxen in einem siebentägigen Kurs des Schweizer MPA-Verbands (SVA) geschult, um dann mit Hilfe eines Ampelschema-Tools weitgehend selbständig Routine-Follow-ups bei Diabetikern durchzuführen [9]. Neben der Aufwertung der MPA durch diese Monitoring-Funktion erfuhr der Hausarzt eine Entlastung von Routinearbeiten. Durch das Ampelschema, das eine automatische Befundinterpretation ermöglichte, wurde sichergestellt, dass auffällige Befunde (gelb und rot kodiert) sofort beim Arzt landeten.

Die Daten der Abschlusserhebung sind derzeit zur Publikation eingereicht. Die Hypothese, dass dieser teamorientierte Ansatz die Versorgung signifikant verbessert, konnte bestätigt werden. Dies ist umso bemerkenswerter, als bereits die Baseline-Erhebung zeigte, dass Diabetespatienten in Schweizer Hausarztpraxen gerade im internationalen Vergleich sehr gut eingestellt sind und z. B. beim HbA1c im Mittel nur noch marginales Potential zur Verbesserung besteht. Ebenso bemerkenswert und erfreulich ist die Tatsache, dass sich für diese aufwendige Studie ausreichend Hausärzte zur Verfügung stellten. Zudem war bis zum Studienende auf Praxisebene kein Dropout zu verzeichnen, d.h. alle Praxen waren bis zur Schlusserhebung dabei. Ein Grund dafür mag auch sein, dass es in allen Projekten üblich ist, nach der Abschlusserhebung die Intervention, also in den genannten Fällen etwa die Kameras sowie die Hausarztund MPA-Schulungen, immer auch der Kontrollgruppe zukommen zu lassen. Dies ist insbesondere für die Motivation der Kontrollgruppe wichtig, ist es doch ein wenig frustrierend, wenn man hochmotiviert an einem Projekt teilnehmen möchte und einen dann der Zufall zum «Stillhalten» verurteilt.

\section{Schweizer Hausärzte sind motiviert, sich an Forschung zu beteiligen}

Es zeigt sich also: Schweizer Hausärzte sind sehr wohl motivierbar, sich auch an aufwendigen Studien zu beteiligen. Es liegt in der Verantwortung der Forscher, entsprechend relevante Fragstellungen zu formulieren und den Aufwand für die beteiligten Ärzte auf das unabdingbare Minimum zu beschränken. Es ist Ausdruck einer eigenständigen Profession, sich durch fachspezifische Forschung an veränderte gesellschaftliche Rahmenbedingungen anzupassen und sich dabei auch immer wieder neu und klar zu definieren. Die Erfahrungen des Institutes für Hausarztmedizin in Zürich zeigen, dass viele Hausärzte dies erkannt haben und sich mit teilweise bewundernswertem Engagement an der Gestaltung und Weiterentwicklung ihres Fachgebiets beteiligen. Hausärzte, die sich an Versorgungsforschung beteiligen, leisten somit nicht nur einen Beitrag für ihre eigene Profession, sie helfen auch mit, das Gesundheitssystem langfristig ressourceneffizient auszurichten. In $\mathrm{Zu}$ kunft wird es daher wichtig sein, ihren Aufwand für die Forschung auch finanziell angemessen abzugelten. Bis dahin gebührt diesen Kolleginnen und Kollegen umso mehr Dank und Anerkennung!

\section{Literatur}

1 Rosemann T, Szecsenyi J. General practitioners' attitudes towards research in primary care: qualitative results of a cross sectional study. BMC Fam Pract. 2004;5(1):31.

2 Chmiel C, Bhend H, Senn O, Zoller M, Rosemann T; FIRE study-group. The FIRE project: a milestone for research in primary care in Switzerland. Swiss Med Wkly. 2011;140:w13142. doi: 10.4414/smw.2011.13142.

3 Tandjung R, Senn O, Rosemann T, Loy M. Diagnosis and management of acute coronary syndrome in an outpatient setting: good guideline adherence in Swiss primary care. J Eval Clin Pract. 2012; May 8. doi: 10.1111/j.1365-2753.2012.01856.x. [Epub ahead of print].

4 Eichler K, Imhof D, Chmiel C, Zoller M, Senn O, Rosemann T, et al. The provision of out-of-hours care and associated costs in an urban area of Switzerland: a cost description study. BMC Fam Pract. 2010;11:99.

5 Steurer-Stey C, Zoller M, Chmiel Moshinsky C, Senn O, Rosemann T. Does a colour-coded blood pressure diary improve blood pressure control for patients in general practice: the CoCo trial. Trials. 2010;11:38.

6 Badertscher N, Rosemann T, Tandjung R, Braun RP. minSKIN does a multifaceted intervention improve the competence in the diagnosis of skin cancer by general practitioners? Study protocol for a randomised controlled trial. Trials. 2011;12:165

7 Frei A, Chmiel C, Schläpfer H, Birnbaum B, Held U, Steurer J, Rosemann T. The Chronic CARe for diAbeTes study (CARAT): a cluster randomized controlled trial. Cardiovasc Diabetol. 2010;15;9:23.

8 Frei A, Herzog S, Woitzek K, Held U, Senn O, Rosemann T, Chmiel C. Characteristics of poorly controlled Type 2 diabetes patients in Swiss primary care. Cardiovasc Diabetol. 2012;11(1):70. [Epub ahead of print].

9 Chmiel C, Birnbaum B, Gensichen J, Rosemann T, Frei A. The diabetes traffic light scheme - development of an instrument for the case management in patients with diabetes mellitus in primary care. Praxis (Bern 1994). 2011; Nov 30;100(24):1457-73. 